contributing with 3000 persons and 200 exposure measurements, 7000 persons and 1000 exposure measurements, 7000 persons and 500 exposure measurements, respectively. From the employment records, individual personal id-numbers, date of birth, sex, first and last date of job specific employment periods are extracted. The cohort will be linked to our national Causes of Death register. Cause of death will be established according to ICD 6-10 and compared to national and if possible regional registers. We will establish a measurement database and a site questionnaire for each company. In our analysis we will investigate the total and cause-specific mortality experience of current and former workers as compared to corresponding national and regional populations and internally-derived control groups, with adjustment for potential confounding factors and focus on lung cancer; characterise the past and current working environment of subjects relative to work area, job title/function and potential for exposure to WCCo, as well as the component exposures: tungsten, tungsten carbide (without cobalt), carbon black, and cobalt; and determine the relationship between level and duration of exposures and lung cancer mortality with adjustment for potential co-exposures, including information obtained on tobacco smoking habits via a nested case-control study.

The epidemiological and exposure assessment components of the study are coordinated by the University of Pittsburgh and the University of Illinois at Chicago, respectively. The study is funded by the International Tungsten Industry Association. Progress in the Swedish studies will be reported separately by us.

\section{OCCUPATIONAL EXPOSURE TO AROMATIC AMINES AND POLYCYCLIC AROMATIC HYDROCARBONS AND BLADDER CANCER: RESULTS FROM THE EPIC COHORT}

${ }^{1} \mathrm{~K}$ G Gawrych, 'Pesch, 'Rabstein, 'Weiss, 'Casjens, 'Rihs, ${ }^{1}$ Angerer, ${ }^{2}$ Bueno-de-Mesquita, ${ }^{2}$ Ros, ${ }^{3}$ Kaaks, ${ }^{3}$ Chang-Claude, ${ }^{4}$ Tjønneland, 'Brüning, ${ }^{5}$ Vineis. ${ }^{1}$ Institute for Prevention and Occupational Medicine of the DGUV (IPA), Bochum, Germany; ${ }^{2}$ National Institute of Public Health and the Environment (RIVM), Bilthoven, The Netherlands; ${ }^{3}$ German Cancer Research Center, Heidelberg, Germany; ${ }^{4}$ Danish Cancer Society Research Centre, Copenhagen, Denmark; ${ }^{5}$ mperial College, London, United Kingdom

\subsection{6/oemed-2013-101717.62}

Objectives Exposure to aromatic amines (AA) has been strongly linked to bladder cancer. Also polycyclic aromatic hydrocarbons (PAH) have been associated with bladder cancer. Here we investigate the bladder cancer risk of occupational exposure to AA and PAH using a large prospective cohort in European populations.

Methods We analysed the bladder cancer risk of exposure to AA and PAH in 754 cases and 833 controls nested in the European Prospective Investigation into Cancer and Nutrition (EPIC) cohort. A semi-quantitative expert rating of the probability and intensity of exposure to $\mathrm{AA}$ and $\mathrm{PAH}$ was performed for 52 occupations known to entail a cancer risk. For each occupation, we multiplied probability $(0=$ none, $1=$ low, $2=$ medium, 3 $=$ high $)$ and intensity $(0=$ none, $1=$ low, $2=$ medium, $3=$ high) of exposure according to an approach for the association with lung cancer. We categorised the subject's sum of scores over all at-risk occupations as low, medium, or high using the tertiles of the distribution in exposed controls. Odds ratios (ORs) with 95\% confidence intervals (CI) were estimated with unconditional logistic regression adjusted for the matching factors and smoking.
Results High occupational exposure to AA and PAH were associated with an estimate of the relative bladder cancer risk of 1.37 (95\% CI 1.02 to 1.84 ) and 1.50 (95\% CI 1.09 to $2.05)$, respectively. We further observed an OR of $1.53(95 \%$ CI 1.03 to 2.28) for ever being exposed to dyestuffs. Ever working in transportation and welding was associated with an increased bladder cancer risk in men that became non-significant after controlling for multiple testing. Smoking and PAH exposure yielded a joint effect of $3.48 \quad(95 \%$ CI 2.51 to 4.84).

Conclusions We were able to confirm the bladder cancer risk associated with aromatic amines and dyestuffs in this large population-based cohort among Europeans. These results provide further evidence that occupational exposure to PAH could entail a bladder cancer risk.

\section{ASBESTOS LUNG BURDEN AND MESOTHELIOMA RISK DUE TO PAST AND CURRENT OCCUPATIONAL AND ENVIRONMENTAL EXPOSURE CONDITIONS IN GREAT BRITAIN}

${ }^{1}$ Aj Darnton, ${ }^{2}$ Peto, ${ }^{2}$ Gilham, ${ }^{2}$ Rake, ${ }^{1}$ Darnton, ${ }^{1}$ Hodgson, ${ }^{3}$ Burdett. ${ }^{1}$ Health and Safety Executive, Bootle, United Kingdom; ' London School of Hygiene and Tropical Medicine, London, United Kingdom; ${ }^{3}$ Health and Safety Laboratory, Buxton, United Kingdom

\subsection{6/oemed-2013-101717.63}

Objectives To characterise the relationship between mesothelioma risk and asbestos lung burden inGreat Britain. To identify the asbestos lung burdens due to current or recent occupational and environmental asbestos exposures, and estimate consequent mesothelioma risks.

Methods Lung tissue and lifetime occupational histories were obtained from 136 mesotheliomas, 263 lung cancers and a random sample of 130 individuals from the British population obtained from those having surgery for pneumothorax. Asbestos lung burdens by fibre type were estimated by Transmission Electron Microscopy. Odds ratios for mesothelioma were estimated for asbestos lung burden categories by logistic regression using lung cancers as controls; mesothelioma risk was then modelled by adjusting for asbestos-related lung cancer risk among controls. Changes in mesothelioma risk arising from occupational and environmental exposures in the past and more recently were estimated from asbestos lung burdens of pneumothorax patients by period of birth.

Results A linear relationship between mesothelioma risk and lung burden was seen up to 0.5 million fibres per dry gram of lung (mfpg), and a burden of $0.1 \mathrm{mfpg}$ was associated with a lifetime mesothelioma risk of $1.4 \%$. Mean asbestos lung burdens for mesothelioma, lung cancer and pneumothorax cases born $<1965$ were positively correlated with the mesothelioma ORs for job categories from previous analyses, with highest burdens associated with construction jobs. Across all jobs, mean lung burdens were highest for mesothelioma $(0.223 \mathrm{mfpg})$, followed by lung cancer (0.060), pneumothorax cases born $<1965$ (0.045) and finally pneumothorax cases born more recently (0.004).

Conclusions The average lifetime risk of mesothelioma in those born since 1965 is an order of magnitude lower than for those born in earlier decades. Lung burden analyses from a larger sample of pneumothorax patients born since 1965 are needed to more precisely identify recent exposure circumstances that contribute to their residual mesothelioma risk. 